\title{
ChemComm
}

Check for updates

Cite this: Chem. Commun., 2019, 55,8776

Received 12th April 2019,

Accepted 8th May 2019

DOI: $10.1039 / \mathrm{c} 9 \mathrm{cc} 02849 \mathrm{~h}$

rsc.li/chemcomm

\section{Switching the enantioselectivity of nanoporous host materials by light $\dagger$}

\author{
Anemar Bruno Kanj, (D) ${ }^{a}$ Jochen Bürck, (D) ${ }^{b}$ Sylvain Grosjean, (DD ${ }^{c}$ Stefan Bräse ${ }^{\text {cde }}$ \\ and Lars Heinke (D)*a
}

\begin{abstract}
A chiral photoswitchable nanoporous material with remote-controllable enantioselective adsorption capacity is presented. This metal-organic framework possesses both homochiral D-camphoric acid and lightresponsive azobenzene moieties. Although the structure at the chiral moieties is unaffected, the trans-cis-azobenzene-photoisomerization changes the pore environment and, thus, switches the enantioselective adsorption behavior of the homochiral MOF.
\end{abstract}

Chiral structures are a central motif in all biological and many artificial molecules, and their (enantioselective) response is fundamental in medical, pharmaceutical and agricultural chemistry. ${ }^{1-4}$ Directing and switching the chirality are imperative to many chemical processes as well as for controlling the structure and functionality of numerous biological systems and molecular assemblies. ${ }^{5-8}$ In contrast to the chirality control by chemical stimuli, ${ }^{5,8}$ use of light as a stimulus can enable very fast switching over long distances with very high spatial resolution. Thus, dynamic remote control over the chirality by reversible photo-isomerization between different molecular enantiomers presents a major aim in chemistry. Recently, the control over the chirality of metal complexes by unidirectional molecular rotary motors based on overcrowded alkenes has been presented ${ }^{9}$ as well as chiral diarylethene compounds with photoswitchable chirality transfer. ${ }^{10}$ The control over the enantioselectivity in catalysis by light has also been demonstrated using molecular rotors based on overcrowded alkenes ${ }^{11}$ as well as with diarylethene. ${ }^{12}$ The chirality of liquid crystals can also be

\footnotetext{
${ }^{a}$ Karlsruhe Institute of Technology (KIT), Institute of Functional Interfaces (IFG), 76344 Eggenstein-Leopoldshafen, Germany. E-mail: Lars.Heinke@kit.edu

${ }^{b}$ KIT, Institute of Biological Interfaces (IBG-2), 76344 Eggenstein-Leopoldshafen, Germany

${ }^{c} K I T$, Institute of Biological Interfaces 3 - Soft Matter Lab (IBG-3), 76344 Eggenstein-Leopoldshafen, Germany

${ }^{d}$ KIT, Institute of Toxicology and Genetics (ITG), 76344 Eggenstein-Leopoldshafen, Germany

${ }^{e}$ KIT, Institute of Organic Chemistry (IOC), 76131 Karlsruhe, Germany

$\dagger$ Electronic supplementary information (ESI) available: Experimental details and infrared spectra. See DOI: 10.1039/c9cc02849h
}

controlled by chiral molecular photoswitches. ${ }^{13}$ On the other hand, solid materials with remote-controllable chiral properties or porous materials with switchable enantioselective adsorption or separation properties have not yet been presented.

Metal-organic frameworks, MOFs, are a sub-class of crystalline nanoporous materials, composed of metal nodes connected by organic linker molecules. ${ }^{14,15}$ By using chiral linker molecules, chiral MOFs can be prepared. ${ }^{16}$ Due to their large variety and their ability to tune their properties by designing and rationally modifying the components, MOF materials are intensively investigated with the aim of efficient separation of achiral ${ }^{17}$ and chiral ${ }^{18}$ molecules. MOFs with remote-controllable properties can be prepared by incorporating photoswitchable molecules like azobenzene, ${ }^{19-22}$ spiropyran $^{23,24}$ or diarylethene. ${ }^{25-27}$ Photoswitchable MOFs with azobenzene pendant groups ${ }^{22}$ have been used to dynamically control the color, ${ }^{28,29}$ adsorption, ${ }^{30-32}$ diffusion and release properties of the guest molecules ${ }^{33,34}$ and the membrane separation performance. ${ }^{35,36}$

Here, a nanoporous material, that is a pillared-layer MOF thin film, is presented which possesses both photoswitchable and chiral properties, Fig. 1. While the pillar linker, AzoBiPyB $(=(E)-2$ (phenyldiazenyl)-1,4-bis(4-pyridyl)benzene), possesses photoswitchable azobenzene side groups, the layer linker, D-camphoric acid (DCam), is homochiral. As a result, each pore of the pillaredlayer $\mathrm{Cu}_{2}(\mathrm{DCam})_{2}$ (AzoBiPyB) MOF comprises two chiral centers and one photoswitchable moiety. The MOF synthesis is performed in a controlled layer-by-layer fashion resulting in thin surfacemounted MOF films, referred to as SURMOFs. ${ }^{37}$ These thin films enable the homogeneous irradiation of the entire sample, UV-vis and CD transmission spectroscopy as well as loading experiments with a quartz crystal microbalance (QCM). By performing uptake experiments with enantiopure probe molecules, $(S)$ - or $(R)$-phenylethanol, we show that the nanoporous homochiral azobenzeneMOF thin film can be photoswitched between enantioselective and unselective forms.

The $\mathrm{Cu}_{2}(\mathrm{DCam})_{2}$ (AzoBiPyB) SURMOF is grown in a layer-bylayer (lbl) fashion by alternatively exposing the substrate to both synthesis solutions, ethanolic copper(II) acetate and the 


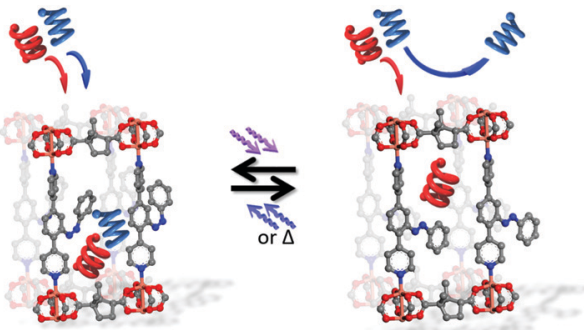

Fig. 1 The structure of SURMOF $\mathrm{Cu}_{2}(\mathrm{DCam})_{2}(\mathrm{AzoBiPyB})$ with the azobenzene side groups in the cis state (left) and in the trans state (right). While absorption of UV light results in trans-to-cis isomerization, blue light absorption or thermal relaxation results in the isomerization back to the trans state. The chiral DCam (layer) linkers horizontally connect the $\mathrm{Cu}$ node. These layers are pillared by the switchable AzoBiPyB linker. The enantioselective adsorption of chiral molecules, simplified as springs, is sketched.

ethanolic linker solution of D-camphoric acid and AzoBiPyB. Between each immersion step, the samples were cleaned with pure ethanol. More details on the SURMOF synthesis can be found in previous publications. ${ }^{37,39} \mathrm{X}$-ray diffractograms, Fig. 2a, show that the sample is crystalline and has the targeted structure. The film is grown in the [001] direction, with the AzoBiPyB SURMOF linker perpendicular to the surface, as pictured in Fig. 1.

UV-vis spectra, Fig. 2b, show that the absorption band at $320 \mathrm{~nm}$, which is correlated with the azobenzene $\pi-\pi^{*}$ transition, decreases upon UV irradiation. The $\pi-\pi^{*}$ band increases to its initial value upon irradiation with blue light $(455 \mathrm{~nm})$ or by thermal relaxation. The absorption band at $450 \mathrm{~nm}$, correlated with the azobenzene $\mathrm{n}-\pi^{*}$ transition, increases upon UV irradiation and decreases again upon blue light irradiation or thermal relaxation. Comparison with the switching of azobenzene in solution $^{40}$ or of the AzoBiPyB linker molecule in solution ${ }^{41}$ is a clear indication for UV-light-induced isomerization from the thermodynamically stable trans state to the excited cis state, while blue light as well as thermal relaxation results in cis-to-trans isomerization. This is in agreement with previous investigations of photoswitchable SURMOFs with the AzoBiPyB-linker. ${ }^{35,42}$ The time constant for thermal cis-to-trans isomerization was determined to be about 3 weeks at room temperature and approximately $10 \mathrm{~h}$ at $50{ }^{\circ} \mathrm{C},{ }^{42}$ which is much longer than the running time of the enantioselective adsorption experiments.

Infrared vibrational spectroscopy, ESI, $\dagger$ Fig. S1, and comparison with ref. 43 and 44 also verify the trans-cis isomerization. This shows that, upon UV irradiation, the maximum yield of $c i s$ azobenzene is approximately $60 \%$ (and $40 \%$ trans), referred to as the cis state. This value corresponds to the switching yield found in similar MOF structures. ${ }^{35,45}$ The maximum amount of trans azobenzene is $100 \%$, obtained upon thermal relaxation. This is referred to as the trans state.

The chiral properties of the SURMOF are investigated by circular dichroism (CD) spectroscopy, Fig. 2c. The CD spectra are virtually identical to the CD spectra of a similar chiral MOF thin film with identical chiral D-camphoric acid linkers. ${ }^{46}$ Moreover, the chiral optical properties of the SURMOF with the azobenzene groups in the trans and in the cis state are essentially identical. Identical CD spectra for trans and cis were expected, since the photoswitchable groups are separated from the chiral D-camphoric acid centers.

The enantiopure uptakes of $(S)$ - and $(R)$-phenylethanol by the homochiral SURMOF in the trans and cis states recorded with a $\mathrm{QCM}^{47}$ are shown in Fig. 3. In the trans-SURMOF, Fig. 3a, the adsorption capacity for $(S)$-phenylethanol is significantly larger than the adsorption capacity for $(R)$-phenylethanol. The average of all uptake runs shows that the uptake of $(S)$-phenylethanol is approximately $2.9 \pm 1.1$ times as large as the uptake of $(R)$-phenylethanol. The enantioselective behavior dramatically changes upon UV irradiation of the $\mathrm{Cu}_{2}(\mathrm{DCam})_{2}-$ (AzoBiPyB) SURMOF sample. In the cis state, the adsorption capacities of $(S)$ - and $(R)$-phenylethanol are very similar and no significant enantioselective behavior was found. On average, the uptake by the cis-SURMOF of (S)-phenylethanol is approximately $1.16 \pm 0.10$ times the uptake of $(R)$-phenylethanol. The summary of the enantioselective behavior is shown in Fig. 4 . The theoretical enantiomeric excesses ee of $(S)$ - versus $(R)$-phenylethanol can be calculated from the enantiopure uptakes, where the interaction between the different enantiomers is neglected, corresponding to the ee at very low concentrations. As a result,
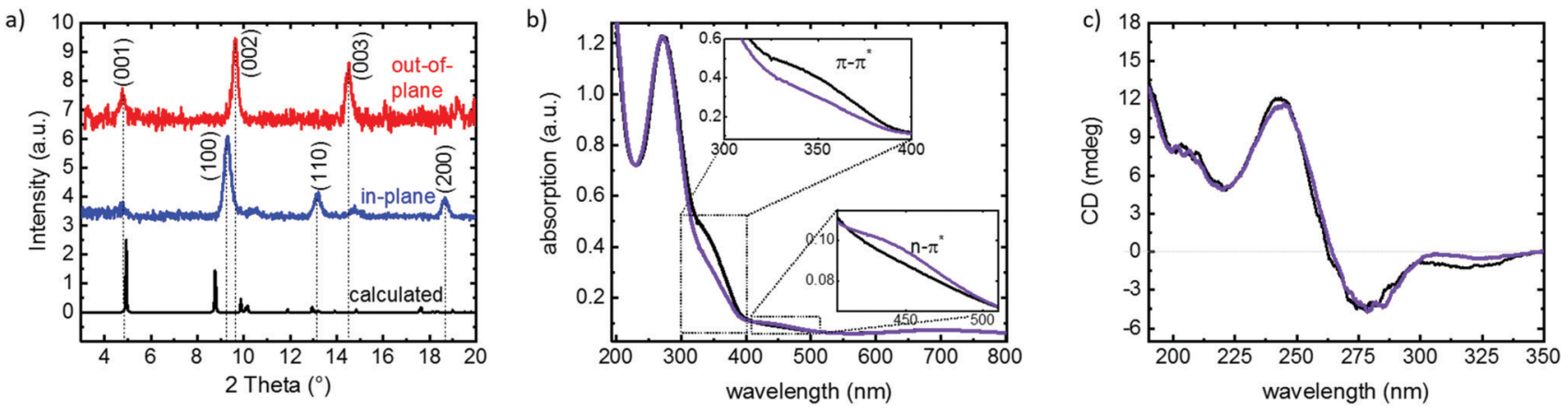

Fig. $2 \mathrm{Cu}_{2}(\mathrm{DCam})_{2}$ (AzoBiPyB) SURMOF characterization. (a) X-ray diffractograms of the sample in the out-of-plane (red) and in-plane (blue) geometry. The calculated diffractogram is shown in black. The crystal planes of the diffraction peaks are labelled. (b) UV-vis spectra of the sample in the thermodynamically stable trans state (black) and upon irradiation with UV light, resulting in the cis state (violet). The $\pi-\pi^{\star}$ and $n-\pi^{\star}$ bands of azobenzene are shown in the insets. The large absorption band at about $270 \mathrm{~nm}$ results from the ligand-to-metal charge transfer at the Cu node. ${ }^{38}$ (c) $\mathrm{CD}$ spectra of the sample in the trans (black) and cis (violet) state. 

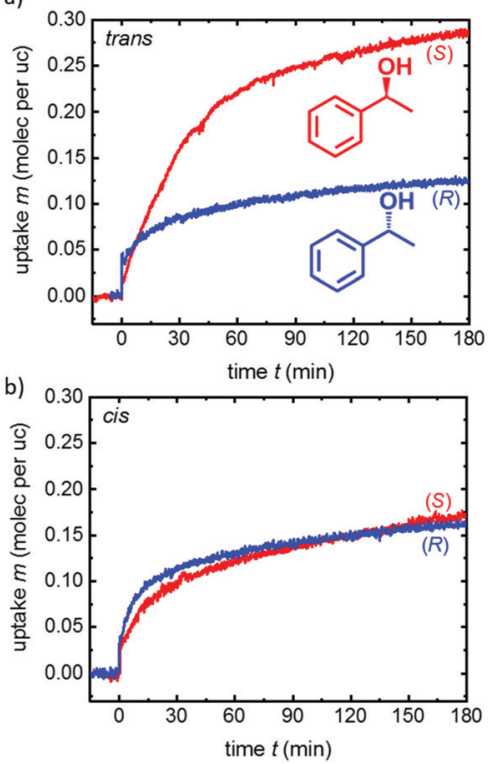

Fig. 3 Enantiopure uptakes of $(R)$ - and $(S)$-phenylethanol by the $\mathrm{Cu}_{2}$ (DCam) $)_{2}$ (AzoBiPyB) film in the trans state (a) and cis state (b). The uptake is determined with a quartz crystal microbalance (QCM) in combination with a gas flow system. The molecular structures of $(R)$ - and $(S)$-phenylethanol are sketched in the inset in (a).

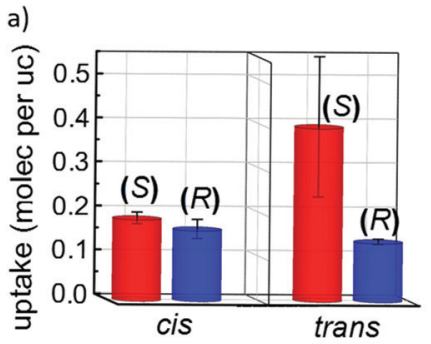

b)

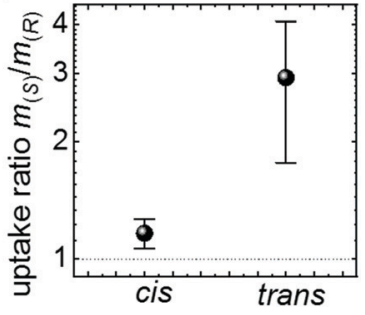

Fig. 4 Enantioselective adsorption. (a) Uptake of (S)-phenylethanol $m_{(S)}$ and $(R)$-phenylethanol $m_{(R)}$ by the homochiral SURMOF in the trans and cis state. (b) Ratio of $m_{(S)}$ and $m_{(R)}$ for the sample in the trans and cis states with the standard deviation as error bars.

while the ee in the cis state is only $7 \pm 4 \%$, the ee in the trans state is $49 \pm 15 \%$.

The enantioselectivity switching effect can be understood on a qualitative level. In the trans state, the nonpolar azobenzene moieties have only minor interaction with the alcohol guest molecules, as found in a previous publication. ${ }^{48}$ The interaction with other parts of the framework prevails, here with the chiral DCam linker molecules. In agreement with $(R) /(S)$-(2)-butanol in camphorate-MOFs, ${ }^{49}$ we assume the formation of H-bonds and dipole-dipole interactions between the guest's OH-group and the host's carboxylate group in proximity to the chiral MOF centers. This causes the observed large enantioselective adsorption of the nanoporous material. In contrast, the attractive interaction between the polar (cis) azobenzene and the polar guest molecules is significantly increased in the cis SURMOF, based on dipoledipole interaction and the formation of $\mathrm{H}$-bonds. ${ }^{48,50} \mathrm{So}$, the guest binds at the azobenzene moiety and not at the chiral center.
Thus, the enantioselective adsorption properties are minute. The fact that the enantioselective response of the MOF material depends not only on the chiral center but also on the pore environment was previously found, where a significant impact of the pore size on the enantioselective adsorption was demonstrated. ${ }^{51}$ It has to be stated that although large efforts have been made, quantum-chemical calculation using both periodic and molecular density functional theory for the simulation of the observed switchable enantioselective adsorption behavior were not successful.

The enantioselective properties of a homochiral nanoporous MOF material significantly depend on the guest-host interaction, which is typically more complex than the simple three-point interaction. ${ }^{52}$ Since clear rules for determining the MOF material properties allowing efficient enantioselective separation of a given pair of chiral molecules, or vice versa, have not yet been presented, the research is often done by trial-and-error. It was found that the enantioselective response of a homochiral MOF material significantly depends on the pair of guest molecules. ${ }^{49,53-55}$ Here, complementary to the experiments with $(R)$ - and $(S)$-phenylethanol, the enantiopure uptakes of less polar $(R)$ - and $(S)$-2octanol by the chiral SURMOF were investigated, Fig. S2 (ESI $\dagger$ ); where there was no large difference found in both between the enantiomers and between the trans and cis forms of the host SURMOF.

In conclusion, a nanoporous thin film is presented which possesses both photoswitchable azobenzene and chiral camphoric acid moieties in the host framework. Uptake experiments with $(S)$ and $(R)$-phenylethanol as probe molecules show that the enantioselective adsorption behavior changes tremendously by the azobenzene photoisomerization - although the chiral centers are not modified. While the MOF thin film with azobenzene in the cis state shows only minute enantioselectivity, the MOF with the azobenzene in the trans state shows strong enantioselective behavior.

The switching of the enantioselective interaction can be extended to materials with chiral azobenzene moieties ${ }^{56,57}$ or photoswitches that can be switched between two chiral forms., ${ }^{9,11}$ Moreover, incorporating such photoswitches in MOF materials with even more complex pore structures and different chiral centers is possible, resulting in switching between different complex stereoselective interactions. We foresee that this concept helps realize efficient enantioselective separation via membranes ${ }^{58,59}$ that allow dynamic switching between racemic mixture and enantioenriched permeates.

We are grateful to the Volkswagenstiftung and the DFG (HE 7036/5) for financial support. We thank Tobias Schlöder and Wolfgang Wenzel (KIT) for their efforts and attempts to quantum-chemically simulate the observed enantioselective adsorption and for fruitful discussions.

\section{Conflicts of interest}

There are no conflicts to declare.

\section{Notes and references}

1 D. W. Green, J.-M. Lee, E.-J. Kim, D.-J. Lee and H.-S. Jung, Adv. Mater. Interfaces, 2016, 3, 1500411. 
2 E. Yashima, K. Maeda, H. Iida, Y. Furusho and K. Nagai, Chem. Rev., 2009, 109, 6102-6211.

3 L. D. Barron, Space Sci. Rev., 2008, 135, 187-201.

4 I. Agranat, H. Caner and A. Caldwell, Nat. Rev. Drug Discovery, 2002, 1, 753-768.

5 H. Miyake and H. Tsukube, Chem. Soc. Rev., 2012, 41, 6977-6991.

6 D. Pijper and B. L. Feringa, Soft Matter, 2008, 4, 1349-1372.

7 J. Crassous, Chem. Soc. Rev., 2009, 38, 830-845.

8 R. M. Meudtner and S. Hecht, Angew. Chem., Int. Ed., 2008, 47, 4926-4930.

9 D. Zhao, T. van Leeuwen, J. Cheng and B. L. Feringa, Nat. Chem., 2016, 9, 250.

10 C. Jurissek, F. Berger, F. Eisenreich, M. Kathan and S. Hecht, Angew. Chem., Int. Ed., 2019, 58, 1945-1949.

11 D. P. Zhao, T. M. Neubauer and B. L. Feringa, Nat. Commun., 2015, 6, 7.

12 D. Sud, T. B. Norsten and N. R. Branda, Angew. Chem., Int. Ed., 2005, 44, 2019-2021.

13 Y. Wang and Q. Li, Adv. Mater., 2012, 24, 1926-1945.

14 H. Furukawa, K. E. Cordova, M. O'Keeffe and O. M. Yaghi, Science, 2013, 341, 1230444.

15 S. Kaskel, The Chemistry of Metal-Organic Frameworks: Synthesis, Characterization, and Applications, Wiley, 2016.

16 G. Nickerl, A. Henschel, R. Grunker, K. Gedrich and S. Kaskel, Chem. Ing. Tech., 2011, 83, 90-103.

17 N. Rangnekar, N. Mittal, B. Elyassi, J. Caro and M. Tsapatsis, Chem. Soc. Rev., 2015, 44, 7128-7154.

18 T. Duerinck and J. F. M. Denayer, Chem. Eng. Sci., 2015, 124, 179-187.

19 C. L. Jones, A. J. Tansell and T. L. Easun, J. Mater. Chem. A, 2016, 4, 6714-6723.

20 O. S. Bushuyev, T. Friscic and C. J. Barrett, CrystEngComm, 2016, 18, 7204-7211.

21 S. Castellanos, F. Kapteijn and J. Gascon, CrystEngComm, 2016, 18, 4006-4012.

22 A. B. Kanj, K. Müller and L. Heinke, Macromol. Rapid Commun., 2017, 38, 1700239.

23 S. Garg, H. Schwartz, M. Kozlowska, A. B. Kanj, K. Müller, W. Wenzel, U. Ruschewitz and L. Heinke, Angew. Chem., 2019, 58, 1193-1197.

24 H. A. Schwartz, S. Olthof, D. Schaniel, K. Meerholz and U. Ruschewitz, Inorg. Chem., 2017, 56, 13100-13110.

25 D. G. Patel, I. M. Walton, J. M. Cox, C. J. Gleason, D. R. Butzer and J. B. Benedict, Chem. Commun., 2014, 50, 2653-2656.

26 I. M. Walton, J. M. Cox, J. A. Coppin, C. M. Linderman, D. G. Patel and J. B. Benedict, Chem. Commun., 2013, 49, 8012-8014.

27 J. Park, D. Feng, S. Yuan and H.-C. Zhou, Angew. Chem., Int. Ed., 2015, 54, 430-435.

28 S. Bernt, M. Feyand, A. Modrow, J. Wack, J. Senker and N. Stock, Eur. J. Inorg. Chem., 2011, 5378-5383.

29 J. S. Caddy, T. B. Faust, I. M. Walton, J. M. Cox, J. B. Benedict, M. B. Solomon, P. D. Southon, C. J. Kepert and D. M. D'Alessandro, Aust. J. Chem., 2017, 70, 1171-1179.

30 A. Modrow, D. Zargarani, R. Herges and N. Stock, Dalton Trans., 2012, 41, 8690-8696.

31 J. Park, D. Q. Yuan, K. T. Pham, J. R. Li, A. Yakovenko and H. C. Zhou, J. Am. Chem. Soc., 2012, 134, 99-102.

32 Y. Zhu and W. Zhang, Chem. Sci., 2014, 5, 4957-4961.
33 J. Brown, B. L. Henderson, M. D. Kiesz, A. C. Whalley, W. Morris, S. Grunder, H. Deng, H. Furukawa, J. I. Zink, J. F. Stoddart and O. M. Yaghi, Chem. Sci., 2013, 4, 2858-2864.

34 L. Heinke, M. Cakici, M. Dommaschk, S. Grosjean, R. Herges, S. Bräse and C. Wöll, ACS Nano, 2014, 8, 1463-1467.

35 Z. Wang, A. Knebel, S. Grosjean, D. Wagner, S. Bräse, C. Wöll, J. Caro and L. Heinke, Nat. Commun., 2016, 7, 13872.

36 K. Müller, A. Knebel, F. Zhao, D. Bléger, J. Caro and L. Heinke, Chem. - Eur. J., 2017, 23, 5434-5438.

37 O. Shekhah, H. Wang, S. Kowarik, F. Schreiber, M. Paulus, M. Tolan, C. Sternemann, F. Evers, D. Zacher, R. A. Fischer and C. Wöll, J. Am. Chem. Soc., 2007, 129, 15118-15119.

38 Z. Gu, L. Heinke, C. Wöll, T. Neumann, W. Wenzel, Q. Li, K. Fink, O. D. Gordan and D. R. T. Zahn, Appl. Phys. Lett., 2015, 107, 183301.

39 L. Heinke and C. Wöll, Adv. Mater., 2019, 1806324.

40 H. M. D. Bandara and S. C. Burdette, Chem. Soc. Rev., 2012, 41, 1809-1825.

41 Z. Wang, L. Heinke, J. Jelic, M. Cakici, M. Dommaschk, R. J. Maurer, H. Oberhofer, S. Grosjean, R. Herges, S. Bräse, K. Reuter and C. Wöll, Phys. Chem. Chem. Phys., 2015, 17, 14582-14587.

42 X. Yu, Z. Wang, M. Buchholz, N. Fullgrabe, S. Grosjean, F. Bebensee, S. Bräse, C. Wöll and L. Heinke, Phys. Chem. Chem. Phys., 2015, 17, 22721-22725.

43 D. Hermann, H. Emerich, R. Lepski, D. Schaniel and U. Ruschewitz, Inorg. Chem., 2013, 52, 2744-2749.

44 L. Duarte, R. Fausto and I. Reva, Phys. Chem. Chem. Phys., 2014, 16, 16919-16930.

45 Z. B. Wang, K. Müller, M. Valasek, S. Grosjean, S. Bräse, C. Wöll, M. Mayor and L. Heinke, J. Phys. Chem. C, 2018, 122, 19044-19050.

46 Z.-G. Gu, J. Bürck, A. Bihlmeier, J. Liu, O. Shekhah, P. G. Weidler, C. Azucena, Z. Wang, S. Heissler, H. Gliemann, W. Klopper, A. S. Ulrich and C. Wöll, Chem. - Eur. J., 2014, 20, 9879-9882.

47 D. Johannsmann, The Quartz Crystal Microbalance in Soft Matter Research, Springer, 2015.

48 Z. Wang, S. Grosjean, S. Bräse and L. Heinke, ChemPhysChem, 2015, 16, 3779-3783.

49 Y. A. Satska, E. A. Mikhalyova, Z. V. Chernenko, S. V. Kolotilov, M. Zeller, I. V. Komarov, A. V. Tymtsunik, A. Tolmachev, K. S. Gavrilenko and A. W. Addison, RSC Adv., 2016, 6, 93707-93714.

50 K. Müller, J. Helfferich, F. L. Zhao, R. Verma, A. B. Kanj, V. Meded, D. Bléger, W. Wenzel and L. Heinke, Adv. Mater., 2018, 30, 1706551.

51 Z. Gu, S. Grosjean, S. Bräse, C. Wöll and L. Heinke, Chem. Commun., 2015, 51, 8998-9001.

52 A. Berthod, Chiral Recognition in Separation Methods: Mechanisms and Applications, Springer Berlin Heidelberg, 2010.

53 F. Song, C. Wang, J. M. Falkowski, L. Ma and W. Lin, J. Am. Chem. Soc., 2010, 132, 15390-15398.

54 J. Zhang, Z. J. Li, W. Gong, X. Han, Y. Liu and Y. Cui, Inorg. Chem., 2016, 55, 7229-7232.

55 S. A. Boer, Y. Nolvachai, C. Kulsing, L. J. McCormick, C. S. Hawes, P. J. Marriott and D. R. Turner, Chem. - Eur. J., 2014, 20, 11308-11312.

56 A. Painelli, F. Terenziani, L. Angiolini, T. Benelli and L. Giorgini, Chem. - Eur. J., 2005, 11, 6053-6063.

57 B. L. Feringa, R. A. van Delden, N. Koumura and E. M. Geertsema, Chem. Rev., 2000, 100, 1789-1816.

58 Z. X. Kang, M. Xue, L. L. Fan, J. Y. Ding, L. J. Guo, L. X. Gao and S. L. Qiu, Chem. Commun., 2013, 49, 10569-10571.

59 K. Huang, X. Dong, R. Ren and W. Jin, AIChE J., 2013, 59, 4364-4372. 